\title{
INSTALAÇÃO E RESULTADOS PRELIMINARES DE PROGRAMA DE RASTREAMENTO POPULACIONAL DE CÂNCER COLORRETAL EM MUNICÍPIO BRASILEIRO
}

\author{
Screening of colorectal cancer in a brazilian town - preliminary results \\ Rodrigo Oliva PEREZ, Igor PROSCURShIM, Guilherme Pagin São JULIÃo, Mauro PICOLO, \\ Joaquim GAMA-RODRIGUES, Angelita HABR-GAMA
}

ABCDDV/582

Perez RO, Proscurshim I, Julião GPS, Picolo M, Gama-Rodrigues J, Habr-Gama A. Instalação e resultados preliminares de programa de rastreamento populacional de câncer colorretal em município brasileiro. ABCD Arq Bras Cir Dig 2008;21(1):12-5

RESUMO - Racional - O câncer colorretal é causa de morte de cerca de 500.000 pessoas-ano em todo o mundo, sendo a terceira principal causa de óbito por neoplasia. A mortalidade destes pacientes está diretamente relacionada com o estádio em que é feito o diagnóstico. O rastreamento leva ao diagnóstico precoce, sendo uma forma eficaz de diminuir a mortalidade. Objetivo - Estimar qual o efeito de uma campanha de prevenção do câncer colorretal em um município brasileiro, estimar a incidência da doença e antever redução da incidência de CCR a longo prazo. Métodos - O rastreamento consistiu de em realizar o teste de sangue oculto do tipo imunológico $\left(\right.$ Hemosure $^{\circledR}$ ), em toda população acima de 40 anos que estivesse assintomática e que nunca tivesse sido rastreada para câncer colorretal. As pessoas que apresentassem resultado negativo eram cadastradas para exame de controle anual. Já os com positivo eram encaminhados à colonoscopia para avaliar a presença de lesões neoplásicas ou pré-neoplásicas e se presentes seguiam para tratamento. Todos os pacientes deveriam ser seguidos por período de pelo menos 10 anos. Durante quatro dias esteve exposto no Ginásio Desportivo Municipal, o "Intestino Gigante" - réplica do cólon humano criado pela ABRAPRECI - informando de forma clara e expositiva à população quais as principais partes e doenças deste órgão, através de alto-falantes embutidos na sua estrutura. O Hemosure ${ }^{\circledR}$ foi o teste de sangue oculto empregado. Pacientes que apresentaram resultados com necessidade de intervenção cirúrgica ou outro tipo de tratamento eram referenciados para centros especializados próximos Resultados - No período de agosto de 2006 a março de 2007 foram entregues 4.567 Hemosure ${ }^{\circledR}$ para pessoas que atendiam os critérios de inclusão. Esse número correspondeu a 54,8\% da população acima de 40 anos do município. Do total, 905 (19,8\%) não foram devolvidos e $22(0,5 \%)$ não puderam ser analisados. Desta forma, 3.640 exames foram analisados, o que corresponde a $43,7 \%$ da população alvo e $79,7 \%$ dos exames distribuídos. Foram obtidos 390 exames com resultado positivo (10,7\%) e 3.250 negativos $(89,3 \%)$. Dos 245 pacientes positivos convocados a realizar colonoscopia, $33(13,5 \%)$ se recusaram. Das 212 colonoscopias realizadas foram diagnosticados: 53 pacientes com doença diverticular, 59 com 1 ou mais pólipos, 9 com adenocarcinomas e 91 colonoscopias normais. Os pacientes com adenocarcinoma, 3 foram tratados endoscopicamente por portarem lesão pequena e precoce, os outros 6 pacientes foram encaminhados para tratamento cirúrgico e quimioterápico. Conclus ões - Os resultados preliminares são insuficientes para estimar qual foi o real efeito da campanha, contudo, pode-se antever redução da incidência de CCR a longo prazo, além de antecipação do diagnóstico e, portanto, do estádio da doença melhorando o prognóstico.

DESCRITORES - Câncer colorretal. Rastreamento. Teste sangue oculto nas fezes.

\section{INTRODUÇÃO}

O câncer colorretal (CCR) é causa de morte de cerca de 500.000 pessoas-ano em todo o mundo, sendo a terceira principal causa de óbito por neoplasia. A taxa de mortalidade destes pacientes está diretamente relacionada com o estádio em que é feito o diagnóstico. O rastreamento leva ao diagnóstico precoce, sendo uma forma eficaz de diminuir a mortalidade. É doença decorrente da degeneração do epitélio colônico, que antes de tornar-se adenocarcinoma em geral surge como lesão pré-maligna, o pólipo adenomatoso 6 . O tratamento adequado para o pólipo consiste na ressecção da lesão - o que na maioria dos casos é feito por via endoscópica -, não sendo necessário ressecção cirúrgica com grandes

Trabalho realizado no município de Santa Cruz das Palmeiras, SP, Brasil.

Endereço para correspondência: Angelita Habr-Gama E-mail: cbcd@cbcd.org.br margens ou linfadenectomia, e nem mesmo tratamento adjuvante ou neoadjuvante com quimiorradioterapia ${ }^{4}$. Ele leva diversos anos para degenerar-se em adenocarcinoma.

Tendo em vista a praticidade em tratar o pólipo colônico, o seu rastreamento também é forma eficaz de diminuir a incidência e, conseqüentemente, mortalidade pelo CCR. O rastreamento é feito principalmente pelo teste de sangue oculto nas fezes 5 . Logo, campanha de rastreamento para CCR na verdade é de prevenção.

Um programa de rastreamento de âmbito populacional deve ser feito através de exame de baixo custo, fácil realização e que se mostre eficaz em selecionar pacientes com maior chance de apresentar a doença pesquisada. $\mathrm{O}$ teste de sangue oculto nas fezes, o mais utilizado, existe em dois tipos: guaiaco e imunoquímico, sendo que, respectivamente, um detecta a atividade da pseudoperoxidase presente no grupo heme da hemoglobina e o outro a proteína globina da hemoglobina humana.

Apesar da colonoscopia e retossigmoidoscopia flexível 
serem os melhores exames para diagnóstico do CCR, não existem estudos mostrando sua eficácia como método para rastreamento populacional ${ }^{1,2,11}$. $\mathrm{O}$ teste de sangue oculto é melhor para a realização de rastreamentos populacionais por ser mais barato, prático, apresentar menores riscos e por ter maior aceitação populacional. Contudo, ele é pouco sensível e específico, sendo necessária a complementação da investigação quando forem positivos, através de exame de observação direta ou endoscópico.

Atualmente um projeto de rastreamento do CCR está em andamento no Estado de São Paulo. AAssociação Brasileira para Prevenção do Câncer de Intestino (ABRAPRECI), organização não-governamental, iniciou esta campanha em 2006. Para isso um município foi selecionado e algumas de suas características foram consideradas fundamentais para o sucesso do projeto. A principal delas era a de que o governo municipal estivesse disposto a participar conjuntamente com a ABRAPRECI, disponibilizando recursos humanos e instalações para a mobilização populacional. O município selecionado foi Santa Cruz das Palmeiras. Não bastando apenas a boa vontade da Prefeitura Municipal, foi considerado fator importante na escolha pela cidade, a forma como o sistema de saúde estava organizado. O Programa Saúde da Família que vem sendo implantado em diversos municípios brasileiros está já parcialmente implementado lá, onde cerca de $65 \%$ da população é atendida por ele, através dos postos de saúde referenciados e equipe multiprofissional. Adicionalmente, os agentes comunitários acompanham os pacientes com maior freqüência, inclusive com visitas domiciliares, o que propiciaria grande ajuda no decorrer da campanha.

Assim, este trabalho tem por objetivos estimar qual o efeito de uma campanha de prevenção do CCR em um município brasileiro, estimar a incidência da doença e antever redução da incidência de CCR a longo prazo.

\section{MÉTODOS}

A população do município em 2006 era de 28.318 habitantes dentre os quais 8.337 com mais de 40 anos $^{1}$. Os habitantes migrantes são pouco freqüentes tornando possível seguimento mais longo.

O rastreamento consistiu em realizar o teste de sangue oculto do tipo imunológico (Hemosure ${ }^{\circledR}$ ), em toda população acima de 40 anos que estivesse assintomática e que nunca tivesse sido rastreada para CCR. Sua realização não necessitou de dieta prévia a coleta. Aquelas pessoas que apresentassem resultado negativo eram cadastradas para exame de controle anual. Já os com exame positivo eram encaminhados à colonoscopia para avaliar a presença de lesões neoplásicas ou pré-neoplásicas e, se presentes, seguiam para tratamento. Todos os pacientes deveriam ser seguidos por período de pelo menos 10 anos.

A adesão à campanha foi estimulada através de diversas reuniões junto às autoridades locais para determinar qual seria a melhor estratégia para chamar a atenção da população; uso de faixas expostas na cidade; anúncios no rádio, televisão e jornais impressos; contato com lideranças religiosas; divulgação em escolas; utilização da própria equipe sanitária e de saúde do município para divulgação nos postos de saúde

Durante quatro dias esteve exposto no Ginásio Desportivo Municipal, o "Intestino Gigante" - réplica do cólon humano criado pela ABRAPRECI - informando de modo claro e expositivo à população quais as principais partes e doenças deste órgão, através de alto-falantes embutidos na sua estrutura.

Um vídeo explicativo sobre o cólon, o CCR e sua profilaxia foi também apresentado à população antes de ingressarem no Intestino Gigante. Diversos voluntários foram mobilizadas durante esses quatro dias com o intuito de esclarecer importância desta campanha. Equipe multiprofissional de saúde e estudantes de medicina eram orientadores de dúvidas e responsáveis pela distribuição dos testes de sangue oculto para as pessoas que se incluíam dentro do grupo populacional a ser rastreado.

Logo após a visita por dentro do Intestino Gigante, as pessoas com idade maior que 40 anos eram encaminhados a sessões em que profissionais da saúde realizavam um questionário, que de forma ampla analisava alguns hábitos relacionados com risco aumentado para CCR como: tabagismo, etilismo, sedentarismo e dieta; também neste questionário era feita a seleção fina de quais pessoas estavam indicadas a fazer o rastreamento, sendo excluídas aqueles que eram sintomáticos, isto é, que apresentavam sangramento anal ativo, ou aqueles que tinham realizado colonoscopia em um intervalo menor do que três anos, e também as pessoas que tinham antecedente pessoal de CCR.

O Hemosure era apresentado em kit para coleta do exame e também para análise do resultado, não necessitando de laboratórios especializados para isto. A equipe de saúde dos postos de saúde foi treinada para analisar os exames e desta forma o resultado era prontamente adquirido.

Nos quatro meses que seguiram a exposição do Intestino Gigante a entrega de exames foi realizada de forma passiva. Os pacientes que passavam em consulta pelo posto de saúde central ou que procuravam diretamente pelo exame, o recebiam junto às devidas orientações para sua coleta, importância de realização e o papel que cada munícipe tinha em divulgar a campanha.

Nos três meses seguintes, mudou-se para forma ativa. Os exames eram levados casa a casa para a população. Durante os finais de semana, a equipe local de enfermeiros e agentes comunitários foi convidada a participar deste "mutirão". Junto a eles, a equipe da ABRAPRECI se prontificou a também participar, enviando semanalmente médicos e universitários para junto aos agentes comunitários distribuir os kits de exame de casa a casa. Durante a semana, os agentes comunitários levavam os envelopes de exame a todas as casas que rotineiramente visitavam. Todos os quatro postos de saúde da cidade foram envolvidos nesta etapa da campanha e ao final dos três meses todas as casas da zona urbana foram visitadas. Os pacientes com resultado negativo eram orientados a realizar o exame novamente no próximo ano. Já os positivos eram convocados para a próxima etapa do rastreamento, isto é, a realização de colonoscopia.

Pacientes que apresentaram resultados com necessidade de intervenção cirúrgica ou outro tipo de tratamento eram 
referenciados para centros especializados próximos à Santa Cruz (Ribeirão Preto e Campinas).

\section{RESULTADOS}

No período de agosto de 2006 a março de 2007 foram entregues $4.567 \mathrm{Hemosure}^{\circledR}$ para pessoas que atendiam os critérios de inclusão. Esse número correspondeu a $54,8 \%$ da população acima de 40 anos. Do total, 905 (19,8\%) não foram devolvidos e $22(0,5 \%)$ não puderam ser analisados, pois o material não tinha sido corretamente coletado. Desta forma, 3.640 exames foram analisados, o que corresponde a $43,7 \%$ da população alvo e 79,7\% dos exames distribuídos. Foram obtidos 390 exames com resultado positivo $(10,7 \%)$ e 3.250 negativos $(89,3 \%)$.

Dos 245 pacientes positivos convocados a realizar colonoscopia, $33(13,5 \%)$ se recusaram. Das 212 colonoscopias realizadas foram diagnosticados: 53 pacientes com doença diverticular, 59 com um ou mais pólipos (76 adenomatosos de baixo grau, quatro adenomatosos de alto grau e 21 hiperplásicos), 9 com adenocarcinomas e 91 colonoscopias normais. Quatorze biópsias foram negativas, ou seja, com infiltrado inflamatório ou apenas mucosa com edema.

Os pacientes com adenocarcinoma, três foram tratados endoscopicamente por tratar-se de lesão pequena e precoce (1 polipectomia e 2 mucosectomias), os outros seis pacientes foram encaminhados para tratamento cirúrgico e quimioterápico.

\section{DISCUSSÃO}

Como esperado, o resultado positivo geralmente era recebido de forma apreensiva pelas pessoas que não entendiam exatamente o significado do teste de sangue oculto positivo. Com o intuito de diminuir a ansiedade gerada pelo resultado, ele era informado não apenas pelo correio, mas sempre que disponível, também por contato telefônico. Assim, as dúvidas eram sanadas o quanto antes, diminuindo a expectativa de cada indivíduo.

As pessoas com resultado positivo eram convocadas primeiramente para uma explicação coletiva. Grupos de cerca de 60 pessoas recebiam juntas, informações sobre o real significado deste resultado, tiravam todas as dúvidas e também eram, novamente, alertadas da necessidade de realizar colonoscopia, com o objetivo de esclarecer qual o foco de sangramento. Aqueles pacientes que concordavam em realizá-la recebiam neste momento orientações sobre o preparo, assim como a medicação e já era agendado o dia do exame.

As colonoscopias foram realizadas na Santa Casa de Misericórdia por uma equipe de colonoscopistas, sendo o equipamento utilizado todo emprestado por empresas especializadas na comercialização de colonoscópios. Diversos profissionais estiveram envolvidos, desde médicos, enfermeiros, auxiliares de enfermagem e acadêmicos de medicina. No hospital duas salas eram usadas para realização do procedimento, e uma terceira para recuperação póssedação. Os exames eram feitos em regime ambulatorial. Contudo, os pacientes com co-morbidades graves fizeram-no em regime de internação no mesmo hospital, onde ficaram durante o preparo e a recuperação pós-exame. Os laudos dos exames eram feitos imediatamente e entregues aos pacientes, produtos de biópsias e ressecções foram enviadas ao laboratório de anatomia patológica, onde eram processados e os resultados encaminhados aos pacientes por intermédio da ABRAPRECI.

Interessante de se comentar é que o volume de colonoscopias executadas foi o maior em períodos equivalentes no município até então.

Nos primeiros quatro dias de campanha, 961 envelopes de Hemosure ${ }^{\circledR}$ foram entregues à população para iniciar o rastreamento pela pesquisa de sangue oculto nas fezes. Contudo, com a grande queda de entrega passiva nos dias que se sucederam, houve por bem necessidade de mudar a estratégia para busca ativa que deu bom resultado, apresentando números aí então expressivos, ou seja, em três meses 3.520 exames foram entregues a população. Assim sugere-se para novas campanhas que ambas as formas sejam utilizadas.

Várias coortes submetidas ao teste de sangue oculto nas fezes relatadas na literatura resultaram em diminuição na mortalidade por câncer colorretal em países da América do Norte e Europa. Uma meta-análise de quatro grandes estudos controlados, que utilizaram teste de sangue oculto nas fezes de guáiaco bianual, incluindo um total de 350000 pessoas mostrou uma redução da mortalidade por CCR de 14\% (variando de 15\% a $33 \%)^{8}$. Destes, apenas três estudos eram randomizados ${ }^{8,9,10}$. Neste estudo ainda é difícil de estimar a redução da mortalidade, tendo em vista que apenas uma série de exames foi realizada.

As taxas de adesão ao rastreamento variaram de 59,6\% a $89,9 \%$ no primeiro exame e de $38-60 \%$ no último exame da série do rastreamento ${ }^{8}$. Em Santa Cruz das Palmeiras, a taxa de adesão ao primeiro exame da série foi de $79,7 \%$, valor este dentro da faixa esperada. Estes estudos apresentaram uma taxa de positividade do exame de $9,8 \%{ }^{10}, 1,5 \%{ }^{7} ; 1,3 \%^{9}$ e $1,5 \%{ }^{3}$ sendo que foram submetidos à colonoscopia 28, 2, 1 e 1,4\% dos participantes respectivamente. Nesta meta-análise, foi constatado que a incidência do CCR não apresentou diferença entre os indivíduos submetidos ao programa de rastreamento e o grupo controle durante o período do rastreamento. Entretanto, a incidência foi $16 \%$ menor no grupo do rastreamento no período de 5-7 anos após o término do programa de rastreamento.

Em Santa Cruz das Palmeiras, a taxa de positividade foi de $10,7 \%$, valor este acima do normalmente observado ${ }^{3,7,9,10}, 5,8 \%$ dos participantes foram submetidos à colonoscopia e a incidência de CCR diagnosticado em Santa Cruz através da campanha de rastreamento foi de 2,47 casos / mil pessoas ano, valor acima do observado em outro estudo, onde a incidência ano de todas as pessoas que participaram do rastreamento foi de 1,49 casos $/$ mil pessoas ano ${ }^{7}$. Contudo o valor apresentado refere-se a incidência observada ao longo de todo o rastreamento, o qual teve uma duração maior que 10 anos e um tempo seguimento médio de 7,8 anos, o que certamente relaciona-se a menor incidência quando comparado a Santa Cruz das Palmeiras.

Em virtude das diversas doações de materiais, por diversas empresas, à campanha de rastreamento de CCR em Santa Cruz das Palmeiras a análise do real custo da campanha torna-se impreciso. Sabe-se que o custo para o tratamento do CCR quando em estadio avançado é elevado, isto em função do próprio tratamento, justificado por longas cirurgias e longos 
períodos de internação tanto em enfermaria quanto UTI, quimioterapia e radioterapia que geralmente são necessários para complementação do tratamento; assim como do período póstratamento, em que muitos pacientes ficam com estomias que requerem cuidados permanentes e gastos constantes. Pode-se também incluir a este custo o período em que estes indivíduos permanecem afastados do trabalho, uma vez que boa parcela dos pacientes com CCR corresponde a população ativa. Já o CCR diagnosticado em fase precoce permite um tratamento muitas vezes endoscópicos, sem necessitar de longas internações ou tratamento adjuvante com quimioterapia e radioterapia. O retorno às atividades geralmente é precoce, diminuindo o custo absoluto e relativo do seu tratamento. Também pode-se antever a redução da incidência de neoplasias, uma vez que pela campanha de rastreamento é feita a prevenção, quando realiza-se a retirada de pólipos pré-neoplásicos, desta forma prevendo uma diminuição de gastos com CCR no futuro.

Campanhas de rastreamento e prevenção de CCR levam a redução da mortalidade relacionada ao câncer em até $33 \%$. Sua realização não envolve grandes recursos tecnológicos e pode ser realizada em qualquer nível de atendimento a saúde, desde o primário em pequenos postos de saúde até grandes hospitais terciários. Tendo em vista sua praticidade de execução e efeito positivo sobre a mortalidade, novas campanhas devem ser estimuladas e a população constantemente orientada sobre a importância de se prevenir.

\section{CONCLUSÃo}

Os resultados preliminares são insuficientes para estimar qual foi o real efeito da campanha, contudo, pode-se antever redução da incidência de CCR a longo prazo, além de antecipação do diagnóstico e, portanto, do estádio da doença melhorando o prognóstico.

\section{AGRADECIMENTOS}

Ao Prefeito Municipal Gilcimar Dantas e Secretário Municipal de Saúde Ilso Cavalheiro de Santa Cruz das Palmeiras - SP; aos membros da ABRAPRECI; aos participantes do Programa de Rastreamento do Câncer Colorretal, todos voluntários; aos acadêmicos em Medicina da Universidade de São Paulo; às empresas WHPM, INC (fornecedora dos kits de pesquisa de sangue oculto), Montag e Olympus (fornecimento de colonoscópios)

Perez RO, Proscurshim I, Julião GPS, Picolo M, Gama-Rodrigues J, Habr-Gama A. Screening of colorectal cancer in a brazilian town - preliminary results. ABCD Arq Bras Cir Dig 2008;21(1):12-5

ABSTRACT - Background - The colorectal cancer accounts for about 500,000 deaths/year worldwide and ranks third in death by neoplasia. Patient mortality is directly related to its stage when diagnosed. Screening allows early diagnosis, reason why it turns out to be an effective tool to reduce mortality. Aims - To assess the impacts of the colorectal cancer prevention campaign in a Brazilian municipality, to estimate the disease occurrence and to forecast reduction of its incidence in the long term. Methods - The Giant Colon, a replica of the human colon, created by ABRAPRECI, was exposed in the local Sports Gymnasium, in order to educate the population on the parts of the organ and the main diseases affecting it. Screening was then performed with the occult blood test kit, $\left(\mathrm{Hemosure}^{\circledR}\right)$, immunological type, in the population over 40 years of age, asymptomatic and without previous CRC screening. People with negative results were registered for annual control and those with positive result were referred to colonoscopy to determine the presence of neoplasic or pre-neoplasic lesions as well as their treatment. All patients were supposed to be followed up for at least 10 years. People needing surgery or other treatment were referred to neighboring specialized centers. Results - From August 2006 to March 2007, 4,567 Hemosure ${ }^{\circledR}$ tests were delivered to people who met the inclusion criteria. This figure corresponded to $54.8 \%$ of the local population over 40 years. Out of this total, $905(19.8 \%)$ were not returned and $22(0.5 \%)$ could not be analyzed. Therefore, 3,640 tests, $43.7 \%$ of the target population, were analyzed, totaling $79.7 \%$ of the tests handed out. Results were positive in $390(10.7 \%)$ exams and negative in $3,250(89.3 \%)$. Out of the 245 patients with positive result and referred to colonoscopy, $33(13.5 \%)$ refused to undergo the exam. The results of the 212 performed colonoscopies were: 53 patients with diverticular disease, 59 with 1 or more polyps, 9 with adenocarcinoma and 91 were normal. Out of the patients with adenocarcinoma, 3 were treated endoscopically since lesions were small and detected at an early stage and the other 6 were referred to surgery and chemotherapy. Conclusion - The preliminary results are not sufficient to estimate the actual impact of the campaign. However, it is possible to count on the reduction of CRC occurrence in the long term as well as better prognostics thanks to early detection and staging of the disease.

HEADINGS - Colorectal cancer. Screening. Fecal occult blood test.

\section{REFERÊNCIAS}

1. Almeida FFN, Araujo SEA, Santos FPS, et al. Colorectal cancer screening. Rev Hosp Clín Fac Med S Paulo 2000;55(1):35 - 42.

2. Estimativa do Ministério da Saúde para o município de Santa Cruz das Palmeiras - SP. In: www.datasus.gov.br; 2006.

3. Faivre J, Dancourt V, Lejeune C, et al. Reduction in colorectal cancer mortality by fecal occult blood screening in a French controlled study. Gastroenterology 2004;126(7):1674-80.

4. Habr-Gama A, Gama-Rodrigues JJ, Alves PA, Verani E. Colonoscopic Polypectomy. The American Journal of Gastroenterology 1977;68(6):535 - 41.

5. Habr-Gama A, Moreira H, Freitas IM, et al. Rastreamento do câncer colorretal pela prova do guáiaco modificada: estudo multicêntrico no Brasil. GED 1983;2(2):30 - 6.

6. Habr-Gama A, Verani E, Gama-Rodrigues JJ. Pólipos do intestino grosso considerações gerais e sobre sua participação na gênese do câncer. Rev Hosp Clín Fac Med S Paulo 1977;32(6):370 - 6

7. Hardcastle JD, Chamberlain JO, Robinson MH, et al. Randomised controlled trial of faecal-occult-blood screening for colorectal cancer. Lancet 1996;348(9040):1472-7.
8. Heresbach D, Manfredi S, D'Halluin P N, Bretagne JF, Branger B. Review in depth and meta-analysis of controlled trials on colorectal cancer screening by faecal occult blood test. Eur J Gastroenterol Hepatol 2006;18(4):427-33.

9. Kronborg O, Fenger C, Olsen J, Jorgensen OD, Sondergaard O. Randomised study of screening for colorectal cancer with faecal-occult-blood test. Lancet 1996;348(9040):1467-71.

10. Mandel JS, Bond JH, Church TR, et al. Reducing mortality from colorectal cancer by screening for fecal occult blood. Minnesota Colon Cancer Control Study. N Engl J Med 1993;328(19):1365-71.

11. Seeff LC, Manninen DL, Dong FB, et al. Is there endoscopic capacity to provide colorectal cancer screening to the unscreened population in the United States? Gastroenterology 2004;127(6):1661-9.

Conflito de interesse: não há Fonte financiadora: não há Recebido para publicação em: 11/07/2007 Aceito para publicação em: 17/10/2007 